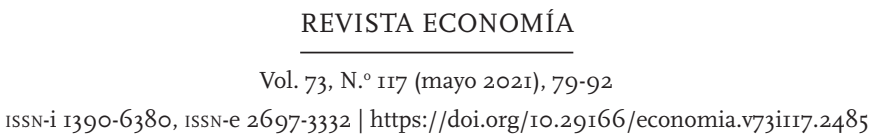

\title{
DINEROS ALTERNATIVOS: HERRAMIENTAS MONETARIAS CON CUALIDAD ECOLÓGICA
}

ALTERNATIVE CURRENCIES: MONETARY TOOLS WITH ECOLOGICAL QUALITY

HÉCTOR LÓPEZ TERÁN

Universidad Nacional Autónoma de México, UNAM (México)

Recepción manuscrito: 20 de agosto de 2020

Aceptación versión final: $1 .^{\circ}$ de abril de 2021

\begin{abstract}
RESUMEN La creación de dinero exponencial producto de la financiarización implica un mecanismo de acumulación de capital que conlleva a constantes crisis económicas y financieras. Ante las constantes crisis, los dineros alternativos han emergido en todo el mundo, desde comunidades locales y actores organizados, como una herramienta monetaria para resolver la escasez de dinero. Si bien, los dineros alternativos coadyuvan a solucionar problemas económicos y sociales, tienen la cualidad de convertirse en una herramienta ecológica al trastocar el esquema de financiarización monetario que dinamiza la reproducción de capital. La propuesta de generar esquemas de economía local empleando el dinero como herramienta para el intercambio, enfrenta la financiarización y el crecimiento exponencial de dinero y de capital que conlleva a una catástrofe ecológica. En este sentido, se consideran que los dineros alternativos empleados con una estructura de producción y consumo orientada hacia la vida asumen una cualidad ecológica.
\end{abstract}

PALABRAS CLAVE Dineros alternativos, financiarización, alternativas al desarrollo

ABSTRACT The exponential money creation product of financialization implies a capital accumulation mechanism that leads to constant economic and financial crises. In the face of constant crises, alternative currencies have emerged throughout the world, from local communities and organized actors, as a monetary tool to solve the currency shortage. Although alternative currencies help to solve economic and social problems, it has the quality of becoming an ecological tool by disrupting the monetary financialization scheme that stimulates the reproduction of capital. The proposal to generate local economic schemes using currency as a tool for exchange faces financialization and the exponential growth of money and capital that leads to an ecological catastrophe. In this sense, alternative currencies used with a production and consumption structure oriented towards life are considered to assume an ecological quality.

KEYWORDS Alternative currencies, financialization, alternatives to development.

Jel CODes A14, B5, E42, P16, Q57. 


\section{INTRODUCCIÓN}

La reproducción de la vida en la modernidad capitalista exige, como mencionó Walter Benjamín, un freno de mano de la locomotora capitalista. La crisis civilizatoria de nuestra sociedad requiere encontrar estrategias múltiples de transformación que trastoquen las estructuras de la reproducción del capital. La imposición y reproducción incesante de la sociedad moderna civilizatoria implica un punto de no retorno para el ser humano y la naturaleza. En las sociedades permeadas por las consecuencias de la crisis civilizatoria multidimensional (Houtart, 2015), surgen estrategias alternativas para vivir otra reproducción de la vida social y enfrentar y resistir la multiplicidad de efectos negativos (Kothari et al., 2019, Lang, König y Regelman, 2019).

Entre las diversas estrategias para construir un modo de vida diferente, en el que se prioriza la vida y no el capital, destacan los dineros alternativos. Los dineros alternativos ${ }^{1}$ son instrumentos monetarios heterodoxos al sistema monetario convencional, contemplan la función del dinero como un medio de intercambio y no de acumulación, surgen para fortalecer la circulación de bienes y servicios en comunidades locales para contrarrestar el sistema económico sustentado en la escasez de dinero y generación de deuda (Schuldt, 1997; Santana Echeagaray, 2011; Corrons 2015, 2017; Lietaer, Kennedy y Rogers, 2015). La circulación de dinero para adquirir bienes y servicios que satisfagan las necesidades individuales genera dinámicas de intercambio local y trastoca las relaciones económicas del mercado capitalista. La crematística aristotélica se diluye ante la cualidad de oxidación monetaria (Gesell, 1936) e impulsa nuevas relaciones sociales con principios de solidaridad, resiliencia y respeto a la naturaleza (Santana Echeagaray, 2011; Acosta, 2013; Brenes, 2013; Corrons, 2017). Los dineros alternativos asumen el dinero como herramienta monetaria de intercambio y no de acumulación para construir nuevas relaciones sociales, enfrentar los efectos negativos de las contradicciones inherentes de la reproducción capitalista y transformar las condiciones económicas, sociales y ambientales de la dinámica de crecimiento exponencial.

En América Latina, diversos proyectos han emergido en contextos diferentes: unos permeados por la tradición ancestral de comunidades indígenas y campesinas, otras por la carencia de dinero circulante y empleo en tiempos de crisis económicas y otras como instrumentos de fomento para mejorar las condiciones económicas y sociales de poblaciones locales (Shephard, 2011; Seyfang y Longhurst, 2012). Aunado a las lógicas de solvencia económica y problemáticas sociales, como puede ser la escasez de empleo, traducida en la carencia de dinero convencional, los dineros alternativos representan una posibilidad para generar alternativas monetarias para enfrentar la dinámica global del metabolismo social de la economía capitalista (Stoffwechsel) (Marx, 2006 [1867], Bellamy, 2000, Schmidt, 2012) mediante estrategias locales que trastoquen la función del dinero valorizado y acumulable por el uso del dinero como herramienta para el intercambio, es decir, el uso del dinero como mercancía que exalte su valor de uso.

En este sentido, el presente trabajo analiza los dineros alternativos como una herramienta monetaria de creación social suscrita a la funcionalidad de satisfacer las necesidades humanas 
como una herramienta monetaria ecológica por la interpelación a las prácticas del metabolismo social capitalista mediante la construcción de dinámicas económicas alternativas desde la economía local. Por lo anterior, el trabajo se desglosa en tres apartados. El primero aborda desde la contradicción fundamental de capital entre valor de cambio y valor de uso- la esencia y la función del dinero convencional como una mercancía utilizada como medio de creación exponencial para la dinámica de acumulación y crecimiento económico que subsume la cualidad instrumental del dinero como medio de intercambio por la cualidad de medio de acumulación y fin último de la sociedad. El segundo apartado realiza una perspectiva panorámica - a grandes rasgos y a lo largo del tiempo- de la emergencia y dilatación de los dineros alternativos como herramientas funcionales para solventar los efectos económicos y sociales de las crisis del capital. El tercer apartado ilustra, a grandes rasgos, el panorama latinoamericano de los dineros alternativos. El último apartado expone a los dineros alternativos como una herramienta monetaria con carácter ecológico para frenar la debacle social y ambiental de la reproducción capitalista mediante la generación de nuevas prácticas de reproducción social en función de escenario resiliente.

\section{LA DOBLE CUALIDAD MERCANTIL DEL DINERO}

El punto inicial de comprender el dinero como una estrategia alternativa parte de desmitificarlo como herramienta circunscrita puramente a la esfera económica y de su doble carácter fetichista que encubre su carácter social y humano (Marx, 2006 [1867]; Harvey, 2015). Antes de mencionar a autores contemporáneos y sus estudios sobre la función y la esencia del dinero, es necesario no olvidar las reflexiones de Silvio Gesell (1862-1930) sobre el dinero libre al proponer utilizar el dinero como un medio de intercambio de circulación plena, con cualidades similares a las demás mercancías, es decir, oxidable y libre de interés (Gesell, 1936). En su obra El nuevo orden económico natural, promueve una reforma monetaria con el objeto de asegurar la circulación efectiva de dinero y eliminar el atesoramiento monetario a partir de su oxidación (Gesell, 1936). Para Gesell (1936), el dinero atesorado debería pagar un tributo con el objeto de aumentar su circulación y disminuir su atesoramiento, asimismo, el dinero debía ser considerado como cualquier otra mercancía. ${ }^{2}$

Existen estudios que abordan el dinero de una perspectiva distinta - al margen del economicismo- que desmitifican la esencia del dinero como un medio de intercambio de carácter social. Por ejemplo, para autores como Greco Jr. (2001), el dinero representa un producto social creado en común acuerdo entre personas por medio del consenso; para Mellor (2017) el dinero representa una construcción social y política de las sociedades, su existencia prescinde del mercado capitalista al estar presente en diversas sociedades previas a la consolidación y desarrollo del capitalismo, desde distintas formas de mercancía ha expresado su valor social por medio del precio, del tamaño de un regalo o de una medida de necesidad (Mellor, 2017). Kennedy (1998) considera que el dinero representa el invento más ingenioso realizado por el ser humano para facilitar el intercambio de bienes y servicios y superar los límites del trueque. ${ }^{3}$ Para Hornborg (2015), el dinero resulta un producto excepcional de la creación humana que puede adquirir diferentes formas y posee una cualidad semiótica, por ello ha tenido diversos 
significados a lo largo de la historia. El dinero, al ser producto histórico, revela su condición de creación en función de necesidades sociales y políticas y no puramente sobre la base de un motivo económico (Polanyi, 2017 [1957]).

La sociedad circunscribe al dinero - como herramienta para el intercambio- en su función de sus valores sociales. El dinero en sí no representa un carácter ético o antiético,${ }^{4}$ sino una herramienta para el intercambio valorizada por la sociedad al otorgarle un carácter o cualidad metafísica a la existencia material y humana, es decir, es un fenómeno social que en la modernidad capitalista su cualidad de objeto deseado se dilata en las relaciones sociales de acumulación de capital (Echeverría, 1991). En el desarrollo del capitalismo, la esencia y función del dinero se trastoca para consolidar la actividad económica del mercado capitalista: medida de valores, medio de circulación, unidad de cuenta y reserva de valor (Marx, 2006 [1867]; Greco Jr., 2001); además, se convierte en un objeto privado y con poder y en el principio y el fin de la sociedad moderna (Kennedy, 1998; Mellor, 2017). El dinero convencional adquiere una forma monopolizada por los bancos centrales, quienes tienen la potestad de emisión de billetes (Lietaer, Kennedy y Rogers, 2015, p. 73), y una capacidad de creación exponencial y sin control por la banca privada (Kennedy, 1998; Greco Jr., 2001). El comportamiento del dinero subyace como una mercancía escasa concentrada en pocas manos dispuesta a ser colocada en manos de quien lo necesita por medio de préstamos y promesas de pago y negarla a quienes no pueden adquirirla. Por eso, el dinero abunda para algunos y escasea para otros. La deuda es el eje rector de las ganancias generadas por el interés propio de la crematística aristotélica.

El proceso de financiarización, la creación del dinero al infinito, representa una separación entre la lógica del dinero y la lógica de producción y la vida humana (Hornborg, 2015; Harvey, 2015). Visto el dinero como mercancía con valor de cambio y valor de uso, la función del dinero en la sociedad capitalista suprime su valor de uso como herramienta para la satisfacción de necesidades y exalta su valor de cambio como mercancía que permite su valorización por medio de sí misma. El asumir ya solo su valor de cambio excluye la esencia de su creación como herramienta destinada al intercambio y su función social de herramienta para satisfacer necesidades. El dinero adquiere la cualidad de creación infinita desfasada de la materialidad de las relaciones de producción que sustentan la reproducción de la vida, crece y se acumula aceleradamente (Hornborg, 2015). La dinámica asumida por el dinero y sus efectos catastróficos constituyen una de las causas para la emergencia de herramientas monetarias alternativas; a lo largo de la historia en diversos momentos críticos de la reproducción capitalista han emergido para solventar las necesidades de la población.

\section{DILATACIÓN DE LOS DINEROS ALTERNATIVOS}

En contraparte al dinero convencional, los dineros alternativos han surgido a lo largo de los años en momentos de emergencia económica producto de las crisis del capital para funcionar como medio de intercambio o instrumento de la socialización de valores de uso que cubren las necesidades de la población, desde sus distintas formas han retomado la esencia y la función del dinero sobre la base de la propuesta de Silvio Gesell (1936). Los llamados dineros de la gente (Lietaer, Kennedy y Rogers, 2015) emergen en núcleos locales donde existen recursos 
disponibles no utilizables, porque no hay dinero, para construir una dinámica de organización y cohesión social y solventar los efectos de la falta de liquidez monetaria y dinamizar la economía local desde la acción de sus propios actores y desde diversas formas: monedas basadas en el tiempo (service credit), sistemas de intercambios mutuos (mutual exchange), monedas locales (local currencies) o mercados de trueque (barler markets) (Schuldt, 1997; Seyfong y Lonhurst, 2013; Hornborg, 2015; Lietaer, Kennedy y Rogers 2015; Corrons, 2017). Los efectos de desigualdad económica y social inherentes al capitalismo permiten la irrupción de alternativas monetarias para generar, desde la organización y participación de actores locales, opciones de intercambio de bienes y servicios para solventar sus necesidades. Ante el control del dinero por el monopolio de la emisión y el sistema financiero privado- buscan construir resiliencia social en la comunidad que adopta un sistema de intercambio alternativo.

Para la economía neoclásica, ${ }^{5}$ este tipo de propuestas de dineros alternativos mencionan Lietaer, Kennedy y Rogers (2015) se pueden considerar como «sistemas insuficientes» (p. 62) porque no se ajustan a la dinámica del mercado global o como un obstáculo al intercambio y la fijación de precios, no obstante, los autores mencionan que «son un accesorio institucional deliberado para separar circuitos de intercambio de distinta naturaleza (locales y de larga distancia) y funciones monetarias diferentes (unidades de valor y medios de pago) (Lietaer, Kennedy y Rogers, 2015, p. 62). Asimismo, el énfasis de los dineros alternativos radica en lo local, su objeto se encuentra en la comunidad. Por otro lado, Hornborg considera que este tipo de alternativas pueden relocalizar el metabolismo social material en las sociedades humanas en el sentido de otorgar a los pobladores la capacidad adquisitiva para consumir bienes y servicios o distribuirlo, por parte del Estado, como un ingreso básico en los hogares (2015).

En este sentido, los dineros alternativos configuran relaciones de confianza, solidaridad y mutuo acuerdo ente los miembros de la red de intercambio (Schuldt, 1997; Lietaer, Kennedy y Rogers, 2015) a través de objetivos, cualidades y necesidades orientados hacia la construcción de nuevas relaciones económicas que buscan el beneficio conjunto de la población creadora. Tejen redes de organización social desde sus actores al involucrarse en las dinámicas de producción, consumo e intercambio para construir un espacio resiliente desde su propias capacidades y para sus propias necesidades por medio de intercambios comerciales locales, sustitución de importaciones y cuidado de la naturaleza y el medio ambiente (Coraggio, 1998; Santana Echeagaray, 2011; Brenes, 2013; Corrons, 2017).

Las prácticas económicas hegemónicas se cuestionan al construir nuevas relaciones sociales y económicas al relegar la crematistica de la económica neoclásica por una economía con prioridad en la reproducción de la vida por encima del capital (Coraggio, 1998; Acosta, 2013). El dinero como mercancía sustentada en su valor de uso adquiere la primicia de solventar las necesidades de emplear una herramienta monetaria para satisfacer otros valores de uso que permitan la reproducción de la vida; el dinero se convierte en un instrumento en función de los seres humanos para vivir y no los seres humanos en instrumentos del dinero. Al trastocar el valor de cambio por valor de uso, el dinero deja de ser funcional para la acumulación y se convierte en un instrumento eficaz para la circulación. En este sentido, son una herramienta que potencializa una alternativa local construida desde sus actores, su territorio y su subjetividad, que desbanca la perspectiva y las relaciones económicas de carácter global por una 
dirigida hacia la comunidad local para contrarrestar sus problemas, potencializar sus capacidades y construir relaciones sociales y económicas alternativas.

En la década de los treinta del siglo pasado, cuando el mundo se encontraba afectado por la crisis económica de la Gran Depresión, en países como Austria, emergía la alternativa del Wörgl como proyecto de dinero alternativo para contrarrestar el desempleo y la escasez de dinero circulante para solventar las necesidades de trabajo y comida de la comunidad: "Alivia la emergencia, da trabajo y pan» (Lindert die not, gibt Arbeit und Brot) se miraba en el encabezado del billete. La experiencia del Wörgl abrió el abanico de innumerables experiencias de dineros alternativos en todo el mundo. ${ }^{6}$ La investigación de Corrons (2017) contabiliza 3418 proyectos en todo el mundo, clasificados en 1715 monedas basadas en el tiempo, 1412 sistemas de intercambios mutuos, 243 monedas locales y 48 mercados de trueques. En Europa, se registran más proyectos con 2333, el 68,3\% de dinero alternativos a nivel mundial, seguido de Asia con el 16,6\%, América del Norte el 9,8\% y América del Sur el 2,7\%, de los cuales la mayoría corresponden a monedas locales y mercados de trueque.

\section{DINEROS ALTERNATIVOS EN AMÉRICA LATINA}

Con los dineros alternativos los sistemas de intercambio funcionan de manera alterna al dinero convencional como herramienta de intercambio con la cualidad de carecer de reconocimiento como moneda de curso legal en tiempos de carencia de circulante en espacios locales necesitados de dinero para dinamizar la economía y la solvencia de la vida de la comunidad (Dittmer, 2015). Entre las distintas funciones del dinero, su esencia subyace en su función como medio de circulación (Kennedy, 1998, Greco Jr., 2001, Corrons, 2017, Mellor, 2017).

Si bien Europa resalta como la principal región con dineros alternativos, en América Latina subyacen proyectos importantes. Empezando de norte a sur, en México la experiencia de dinero alternativo surge desde la década de los noventa del siglo pasado con el proyecto «La otra bolsa de valores» en la que emergió la experiencia del vale multitrueque tláloc utilizado en mercados (tianguis) entre miembros de la red para fomentar un mercado solidario (Santana Echeagaray, 2011). Posteriormente, en 2010, inspirados por la experiencia del tláloc, surgió en el municipio de Espinal, Veracruz la moneda local túmin como instrumento de intercambio entre productores y consumidores, el uso del túmin se ha extendido a varios estados de la República Mexicana (Junta de Buen Gobierno, 2014). Si bien el tláloc y el túmin son las monedas locales de mayor representación en México también destacan los dineros alternativos: amanatli, kuni, fausto, caribe, mixiuhca e itacatate.

En Centroamérica, en Honduras entre 2003 y 2004, con apoyo de la Fundación STRO, se creó dentro de la Red de Comercialización Comunitaria Alternativa (COMAL) la moneda social UDIs (unidades de intercambio solidario), y en 2008, desde la empresa Biocombustibles de Yoro, surgida del proyecto Gota Verde, surgió la moneda PECES para articular a pequeños productores y fomentar la economía local (Shephard, 2011). En Costa Rica, desde la cooperativa Coopevictoria, se crearon las unidades de intercambio solidario (UDIS) sistema de vales para comprar productos de la cooperativa y en una red de comercios locales (Brenes, 2013). En El Salvador, en el municipio de Suchitoto, Cuscatlán, la Cooperativa de Ahorro y Crédito 
ACORG creó las unidades de intercambio solidario suchitotense (UDIS), vales emitidos por la cooperativa, respaldados por garantías y pagarés para otorgar un crédito a tasa de interés bajas a un asociado para que disponga de circulante dentro de la localidad (Buscema, 2010; García Hernández et al., 2013).

En América del Sur, Argentina destaca por la expansión de las redes de trueque en la última década del siglo pasado. En 1995, en Bernal, provincia de Buenos Aires, surgió el Primer Club de Trueque que posteriormente daría inicio a una rápida expansión de experiencias de intercambio solidario por todo el país hasta consolidarse en la Red Global de Trueque. La crisis económica de 2001-2002 sentó las bases para su expansión, la carencia de trabajo y dinero circulante, la contracción en las ventas de productos autónomos y la necesidad de satisfacer necesidades básicas incidieron en la conformación y expansión de las redes de trueque (Hintze et al., 2003), se estimó que para el año 2002 existían alrededor de 2,5 millones de personas y 5000 nodos (Abramovich y Vázquez, 2007). La experiencia argentina es considerada como una muestra relevante de dineros alternativos sujetos a experiencias de economía social y solidaria mediante la agrupación y asociación de actores de la población en nuevas prácticas de integración económica no incorporadas a la economía convencional. Para Abramovich y Vázquez (2007), «la experiencia del trueque muestra que es viable crear mercados y monedas que permitan poner en funcionamiento las capacidades de trabajo y generar nuevos lazos sociales» (pp. 134-135). Actualmente, las redes de trueque han emergido nuevamente como respuesta a los efectos de la crisis económica argentina (Lutzky, 2019).

En Brasil, el Banco Comunitario las Palmas, institución financiera comunitaria en la comunidad de Palmeiras, Fortaleza, Ceará, busca eliminar la pobreza urbana y rural y la reorganización de la economía local por medio de créditos para la producción, el comercio o los servicios e iniciativas enfocadas en la promoción de una economía social y solidaria. Aunado a lo anterior, el banco creó la moneda social palma, moneda complementaria de circulación comunitaria, con el objetivo de subsanar las desigualdades y vulnerabilidades de la población junto a la articulación de redes locales de producción y consumo. La experiencia ha logrado expandir su influencia hacia otras localidades de Brasil con el objetivo de «impulsar la actividad económica en poblaciones marginales» (Corrons, 2017).

En Chile, los vales talentos surgieron en el año 2000 impulsados por el Área de Economía Ecológica del Instituto de Ecología Política como intento de réplica de la Red de Trueque argentina con el objeto de crear un red multitrueque solidaria y recíproca como alternativa a las relaciones económicas de la economía de mercado. En Colombia, ha surgido diversas experiencias de dineros alternativos, uno de los pioneros fue «el bono cafetero» creado en Támara, Casanare, desde la Cooperativa de Caficultores de Támara, como moneda propia que circulaba localmente para fortalecer la economía campesina, el proyecto funcionó por 30 años en coordinación con el Banco del Estado. ${ }^{7}$ Otros ejercicios al margen del Estado son: el altamira surgido en el municipio de Bello utilizado en la urbanización Altamira en 2007; floricambios, en Santa Elena; las monedas utilizadas en ferias: petros, barequeros, ceibas, caciques, ruanas, soles, córdobas; amerrikua en el municipio de Cota, mazorca en el departamento de Caldas y sistemas de trueque en Medellín (Shephard, 2011), y la moneda social la roca. Otros ejercicios de intercambio sin dinero convencional han sido el uso de pasta de coca (base para la creación 
del alcaloide) para la compra de alimentos de la población campesina - un gramo es equivalente a no más de 2000 pesos colombianos- en el pueblo de La Paz, Guaviare. En Venezuela, se crearon en el barrio 23 de Enero, en Caracas, desde el Banco Comunal Banpanal la moneda social los panales.

En países como Bolivia y Ecuador, subyace el intercambio de alimentos desde prácticas ancestrales entre comunidades por medio del trueque entre regiones. Por ejemplo, en Bolivia, en la Isla Suriqui, se intercambian pescado por productos como maíz, frutas y papa; en el pueblo del Mocomoco se intercambia lana y queso del altiplano peruano por maíz y yerbas medicinales; y en las provincias de Saucari, Jacha Carangas y Cercado, se pueden intercambiar diversos tipos de productos como maíz, habas, azúcar, etc. (Medina, 2012). En Ecuador, subyacen prácticas ancestrales de intercambio a través del trueque entre la Costa, la Sierra y la Amazonía. El trueque es una práctica vigente en lugares como Pimampiro e Ibarra, en la provincia de Imbabura (Minadeo, Prada y Fernández-Pacheco, 2014). Además del trueque, en Ecuador han emergido experiencia de dineros alternativos como la Red de Intercambio Mutuo (MUYU), los ecuacanjes y las unidades de intercambio solidario (UDIS) (Minadeo et al., 2014).

En general, entre los distintos ejercicios de dineros alternativos en América Latina, como experiencias efímeras o consolidadas, destacan las monedas sociales y los mercados de trueque. No obstante, la complejidad en sobrellevar el compromiso de una alternativa para perdurar en el tiempo, los diversos ejercicios muestran la propuesta y búsqueda de soluciones alternativas ante las condiciones económicas y sociales de la sociedad latinoamericana en tiempos de crisis. La población vulnerada y excluida económica y socialmente encuentra en estas herramientas una vía para construir un espacio alternativo regido por principios de solidaridad y reciprocidad para satisfacer sus necesidades y generar espacios de cohesión social y resiliencia. Asimismo, la posibilidad de contrarrestar la problemática social permite refuncionalizar paralelamente el dinero desde un carácter ecológico en el sentido de permitir relocalizar los recursos en las personas y la comunidad.

\section{DINEROS ALTERNATIVOS: ALTERNATIVAS ECOLÓGICAS}

Los diferentes ejemplos de dineros alternativos, además que constituir una herramienta económica, logran un alcance ecológico al contemplar el principio de «lo pequeño es hermoso y de las economías de base» (Dittmer, 2015, p. 62), y al ser considerados como una estrategia de desarrollo local sostenible que coadyuvan a detener el deterioro ambiental (Brenes, 2013). Si bien, los dineros alternativos son considerados como herramientas para potenciar el entorno local alrededor de prácticas productivas y consuntivas, uno de los factores principales para considera su carácter ecológico radica en la ruptura con el proceso de financiarización, principal promotor de la destrucción ecológica y del metabolismo social del capital (Bellamy, 2000; Harvey, 2015; Hornborg, 2015).

Entender la dinámica de los dineros alternativos con un carácter ecológico requiere analizar la función del dinero convencional en la dinámica del capital, al constituir el dinero de creación infinita un problema grave para los límites planetarios. La centralidad de la función del dinero en las relaciones de producción y consumo globales representa una contradicción 
con la capacidad planetaria. La creación infinita instaurada desde 1970 con la flexibilización cuantitativa resulta la «caja de Pandora» de creación monetaria para el acelerado proceso de producción y consumo que hoy avanza libre de respaldo físico (Funder y Max-Neef, 2014; Harvey, 2015; Hornborg, 2015).

El dinero sin respaldo atraviesa la vida económica contemporánea, las transacciones realizadas por la liberalización de capitales: industriales, comerciales y financieros conlleva la creación de dinero infinito para el mutuo soporte del capital en conjunto, estimulándose para realizar como fin único: la generación de ganancia. El sistema monetario convencional responde a la dinámica del mercado autorregulado (Polanyi, 2017 [1957]) que impone un metabólico de producción y consumo acelerado de mercancías, al uso del dinero en la mercantilización de todas las esferas de la vida (Harvey, 2007; Echeverría, 2016). El dinero convencional conlleva un carácter insustentable porque funciona para reproducir la lógica de reproducción y consumo de la vida sustentada en el valor de cambio y no contempla la capacidad planeta en la producción acelerada que fundamenta el crecimiento económico.

Ante la situación, los dineros alternativos transitan de la dinámica económica global hacia la dinámica de la económica local. La propuesta de dineros alternativos como estrategias sociales busca tejer en la comunidad nuevas relaciones económicas y sociales a través de (re) funcionalizar el dinero al (re)convertirlo a su esencia instrumental en favor de las personas. Al circunscribir al dinero a una herramienta de intercambio - desprovista de la capacidad de acumulación por la oxidación (Gesell, 1936) - construye prácticas de producción y consumo desde y para su entorno. El dinero se ubica en función de las necesidades de la gente para satisfacer necesidades de reproducción de la vida (Max-Neef, Elizalde y Hopenhayn, 2006) trastocando, en cierta medida, las relaciones estructurales de la reproducción capitalista que suscriben el uso del dinero como bien privado y en función de la acumulación. A diferencia del dinero público - controlado por el Estado- (Mellor, 2017), el dinero local permite una reconfiguración de la organización social por medio de potencializar las capacidades locales; el dinero retorna a su uso como cualquier otra mercancía, asume su carácter servil con motivos y principios propios de aquellos que lo utilizan para solventar la vida.

La adquisición de una práctica con sustrato local permite que las actividades y principios se sustenten en la comunidad, permitiendo mayores escalas de contención ecológica a nivel local y contribuyendo a nivel global. La producción y consumo alternativos y locales permiten generar una aposición a la producción global de mercancías para la satisfacción de necesidades vitales y contribuye a reducir la huella ecológica. Acompañados de esquemas de producción y consumo locales permiten entre los actores involucrados crear dinámicas de satisfacción de necesidades desde sus propias capacidades, generar oportunidades locales que dinamicen el entorno y construyan comunidad. Pueden convertirse en una herramienta de economía local para el desenvolvimiento de actividades alternativas impulsadas por sus actores con la finalidad de potencializar un espacio y en función de sus propias capacidades y necesidades.

Asimismo, es importante reconocerlos como herramientas de transición hacia una economía acorde con la capacidad biofísica del planeta, es decir, acorde con el medio ambiente, porque no puede existir una incompatibilidad entre una vía ecológica de organización social con un esquema monetario sustentado en la creación exponencial. Los dineros alternativos 
asumen el valor de uso de la mercancía dinero para solventar necesidades vitales y delimitan el uso de dinero convencional en aquellas necesidades insolventables dentro de la comunidad; dicha función permite reconfigurar una dinámica del dinero con cualidad ecológica y las dinámicas de producción y consumo de la economía local. No obstante, habría que poner atención en la cualidad de oxidación monetaria (Gesell, 1936) de los dineros alternativos, porque podría integrarse en una dinámica acelerada de producción y consumo que pueden generar un continuo deterioro de la naturaleza. El dinero libre debe estar acompañado de un trastocamiento de la lógica de producción y consumo de la economía convencional, es decir, construir otro metabolismo social en escala local (Toledo, 2013), para poder construir un escenario ecológico diferente, de lo contrario, la libre circulación de dinero para la adquisición de mercancías coadyuvaría a generar un proceso continuo de degradación planetaria similar al de la reproducción capitalista sustentado en el crecimiento económico al infinito.

En este sentido, es necesario enfatizar en un esquema monetario sustentado en la redefinición del dinero desde la creación de los dineros alternativos que retornen el valor de uso del dinero como herramienta monetaria para el intercambio y para la socialización de valores de uso en contrasentido al dinero para la acumulación incesante de capital. El dinero requiere retornar a ser medio y no fin en la vida social. Dittmer (2015) menciona que si se pretende alcanzar otro enfoque que irrumpa en la lógica de crecimiento económico al infinito - subyacente en la reproducción de capital - los dineros alternativos - para responder al problema ecológico - requieren sustentar cuatro aspectos importantes: la consolidación comunitaria, la promoción de valores alternativos, la facilitación de formas alternativas de subsistencia y la ecolocalización. Aunado a lo anterior, es necesario redefinir la función del dinero y la riqueza (Marcellesi, 2008) como una herramientas democratizada para la configuración de economías locales y comunidades de cara a construir un proceso metabólico equilibrado sustentado en las necesidades humanas (Max-Neef, Elizalde y Hopenhayn, 2006) que contrarresten la dinámica productiva y consuntiva acelerada en beneficio de la sociedad y el medio ambiente.

\section{CONCLUSIONES}

El panorama de los dineros alternativos nos muestra la emergencia de iniciativas locales surgidas desde sus propios actores, la manifestación de una iniciativa de alternativa local para resolver problemas que la economía convencional no ha podido solventar. La potencialidad de los dineros locales, radica en la confianza y el compromiso de cada uno de los miembros que integran la red de intercambios, en el compromiso de construir nuevas relaciones sociales y económicas sustentadas en la solidaridad como eje rector primordial. Aunado a eso, devela la esencia del dinero como medio o herramienta económica y no como fin de la vida humana; en su accionar enfrenta la crematística instaurada por la economía convencional.

En tiempo de crisis global de escala civilizatoria, los dineros locales construyen una estrategia alternativa por diferentes aspectos. Primero, genera relaciones de intercambio sustentadas en la satisfacción de las necesidades y el uso potencial de las capacidades. La comunidad y sus individuos contienen la capacidad potencial — desde la base de su experiencia y conocimiento de la vida en comunidad - de aportar con su trabajo un producto o servicio requerido 
por la comunidad local; las necesidades básicas se priorizan más que los principios de acumulación monetaria. Segundo, construyen prácticas de producción y consumo diferentes entre los miembros de la comunidad local en las que instauran la cualidad y la subjetividad de los actores locales, potencializando sus capacidades de acuerdo a sus formas de comprender sus relaciones locales y su territorio; suscriben un modelo de vida desde su contexto local, desde la comprensión de su realidad situada y en orientación prioritaria hacia su comunidad. Y tercero, construyen y fortalecen relaciones entre los miembros de la comunidad; generan cohesión social, puntos de encuentro en la reproducción material de su vida y significaciones manifiestas en sus subjetividades y en la representación de su comunidad.

Los dineros alternativos, además de su potencialidad social, tiene una cualidad ecológica al trastocar y enfrentar el modelo de financiarización, la creación monetaria infinita para la acumulación de capital que conlleva repercusiones ecológicas por la capacidad planetaria finita. Asimismo, su orientación hacia la economía local construye relaciones cercanas de producción y consumo local que pudieran incidir en la huella ecológica al configurar un metabolismo social distinto a escala local. Además, la satisfacción de las necesidades desde la economía local, la cohesión social emanada del uso colectivo e instrumental del dinero y la configuración de una subjetividad individual y colectiva con la comunidad permiten reforzar el propósito colectivo de la reproducción de la vida.

\section{NOTAS}

1 Existe distintas denominaciones sobre este tipo de dinero no convencional: dineros alternativos, porque operan en lugar del dinero convencional; complementarios, porque complementan la dinero convencional; dineros sociales, porque son emitidos y contralados por organizaciones sociales; comunitarios, porque emergen en comunidades para crear un sistema de intercambio alternativo; dinero local, por circular en comunidades locales (Santana Echeagaray, 2011; Lietaer, Kennedy y Rogers, 2015; Corrons, 2017).

2 Para una revisión sobre las distintas concepciones del dinero como mercancía de intercambio (ver Schuldt, 1997).

3 Con la creación del dinero - como medio de circulación - se superaron las barreras del trueque en el que cada transacción necesitaba de la coincidencia de deseos y necesidades de los actores enfrentados en el mercado para el intercambio de equivalentes. Smith (2008) afirmó que las dificultades del trueque permitieron adoptar una mercancía como dinero: «Cuando comenzó a practicarse la división internacional del trabajo, la capacidad de cambio de cambio se vio con frecuencia prohibida y entorpecida en sus operaciones. Es de suponer que un hombre tuviera de una mercancía más de lo que necesitaba, en tanto otro disponía de menos. El primero, en consecuencia, estaría dispuesto a desprenderse del sobrante, y el segundo, a adquirir una parte de este exceso. Mas se acontecía que este último no contaba con nada de lo que el primero había de menester, el cambio entre ellos no podía tener lugar. [...] A fin de evitar inconvenientes de esta naturaleza, todo hombre razonable, en cualquier periodo de la sociedad, después de establecida la división del trabajo, procuró manejar sus negocios de tal forma que en todo tiempo pudiera disponer, además de los productos de su actividad peculiar, de una cierta cantidad de cualquier otra mercancía, que a su juicio escasas personas serían capaces de rechazar a cambio de los productos de sus respectivo esfuerzo» (p. 24).

4 El esquema de valoración instrumental del dinero se distingue de los conceptos de neutralidad o no neutralidad del dinero dentro de la economía desarrollada por distintas escuelas del pensamiento económico (Cruz Hidalgo y Parejo Moruno, 2016). 
5 Desde la economía de mercado han surgido propuestas de monedas competitivas desde la empresa privada como la de Friedrich Von Hayek en su libro La desnacionalización de la moneda (1976). Cabe mencionar que las empresas privadas emplean también mecanismo que funcionan como dineros alternativos para fomentar el consumo y la fidelidad de sus clientes, ejemplo de ello son: los puntos por compras y las millas de viajero frecuente. Subyace aquí una diferencia, mientras que los ejemplos mencionados buscan generar mayor competitividad, clientes, consumo y ganancias dentro del mercado capitalista, los dineros alternativos buscan crear relaciones solidarias y recíprocas en sus relaciones económicas (Santana Echeagaray, 2011).

6 Otros ejemplos de dineros alternativos destacados son: el wära en Alemania, el jak en Dinamarca y Suecia, el wir en Suiza, el paper scrip en EuA. Proyectos más recientes y con mayor impacto y duración son el Local Employment Trade System (LETS) en Canadá y extendido a EUA, el Système d'Échange local (SEL) en Francia, el fureai ticket en Japón, y los time dollars y los Ithaca hours en Estados Unidos (Schuldt, 1997; Shephard, 2011; Lietaer, Kennedy y Rogers, 2015).

7 Si bien este ejemplo es la creación de una herramienta monetaria en favor de los campesinos, se diferencia de aquellas propuestas realizadas desde los actores de la población al margen de las instituciones del Estado.

\section{REFERENCIAS}

Abramovich, A. L. y Vázquez, G. (enero-junio de 2007). Experiencias de la Economía Social y Solidaria en la Argentina. Estudios Fronterizos, 8(15), 121-145.

Bellamy, J. (200o). La ecología de Marx. Materialismo y naturaleza. El viejo topo .

Brenes, E. (2013). Monedas complementarias y ambiente. Cuaderno Interdisciplinario de Desarrollo Sostenible, (10), 111-147.

Buscema, C. (2010). Evaluación de un sistema de moneda complementaria en Suchitoto, El Salvador. (s. d.).

Corrons, A. (junio de 2017). Monedas complementarias: dinero con valores. Revista Internacional de Organizaciones, (18), 119-134.

Cruz Hidalgo, E. y Parejo Moruno, F. M. (2016). El dinero en la historia del pensamiento económico: la teoría monetaria post-keynesiana y su confrontación con la ortodoxia. Iberian Journal of the History of Economic Thought, 27-41.

Dittmer, K. (2015). Monedas sociales. Ecología Polítiica, 62-64.

Echeverría, B. (1991). El dinero y el objeto del deseo. Debate feminista, 155-160.

Echeverría, B. (1998). Valor de uso ontología y semiótica. En B. Echeverría, Valor de uso y utopía (pp. 153-196). Siglo xxi Editores.

Echeverría, B. (2016). Monernidad y blanquitud. Ediciones Era.

Funder, F. y Max-Neef, M. (2014). Local money as solution to capitalist global financial crises. En M. Pirson, U. Steinvorth, C. Largacha-Martínez and C. Dierksmeier, From Capitalistic to Humanistic Business (pp. 157-189). Palgrave-Macmillan.

García Hernández, E., Escoto de Chávez, K., Bustamante Estrada, R. y Turcios Reyes, E. (2013). Evaluación de la moneda alternativa como un medio que posiilita la economía solidaria: El caso de Suchitoto. Revista Realidad, 461-482.

Gesell, S. (1936). El orden económico natural por la libremoneda y libretierra. E. Gesell (ed.).

Greco Jr., T. (2001). Money. Understanding and creating alternatives to legal tender. Chelsea Green Publishing Company.

Harvey, D. (2007). El nuevo imperialismo. Akal . 
Harvey, D. (2015). Diecisiete contradicciones y el fin del capitalismo. Editorial IAEN.

Hintze, S., Federico Sabaté, A. M., Coraggio, J. L. y Cassano, D. (2003). Documento base de la Jornada Nacional sobre Trueque y Economía Solidaria. En S. Hintze, El trueque y la economía solidaria. Universidad Nacional General Sarmiento.

Hornborg, A. (2015). Redesigning money to curb globalization: can we domesticate the root of all evil? Financialisation, economy, society and sustainable development (Working paper series n. ${ }^{\circ} 91$ ).

Houtart, F. (2015). El bien común de la humanidad. Editorial IAEN.

Junta de Buen Gobierno. (2014). Aceptamos Túmin. Mercado Alternativo, Economía Solidaria y Autogestión. CÓDICE/Taller Editorial.

Kennedy, M. (1998). Dinero sin inflación ni tasa de interés. (M. Lambré, H. Heineken, Edits.; B. Romero y H. Heineken, Trads.). Editorial del Nuevo Extremo.

Kothari, A., Salleh, A., Escobar, A., Demaria, F. y Acosta, A. (2019). Pluriverso.Un diccionario del posdesarrollo. Icaria.

Lang , M., König, C.-D. y Regelman, A.-C. (2019). Alternativa en un mundo de crisis. Grupo de trabajo global Más Allá del Desarrollo. Fundación Rosa Luxemburgo, Universidad Andina Simón Bolívar.

Lietaer, B., Kennedy, M. y Rogers, J. (2015). El dinero de la gente: monedas locales y soberanía económica. Icaria.

Lutzky, L. (22 de mayo de 2019). Cómo resurge el trueque en Argentina (o un recuerdo a la crisis de 2001). Rusia Today. https://actualidad.rt.com/actualidad/315570-resurge-trueque -argentina-recuerdo-crisis-2001.

Marcellesi, F. (2008). Sistema de trueque y moneda complementaria en Francia. Ecología Política, 75-77.

Marx, C. (2006 [1867]). El capital. Crítica de la economía política (vol. I). Fondo de Cultura Económica.

Max-Neef, M., Elizalde, A. y Hopenhayn, M. (2006). Desarrollo a escala humana: conceptos, aplicaciones y algunas reflexiones. Icaria Editorial .

Medina, J. (2012). Monedas locales: Una herramienta convivial para facilitar el Vivir Bien. Círculo Achocalla, (4). http://circuloachocalla.org/monedas-locales-una-herramienta-convivial -para-facilitar-el-vivir-bien/\#_ftnrefi.

Mellor, M. (2017). Money for the people. Great transition initiative. Toward a transformative vision and praxis. (s. d.).

Minadeo, G., Prada Araque, D. y Fernández-Pacheco Sáez, J. L. (julio de 2014). Ecuador y los medios de pago complementarios: ¿amenaza u oportunidad? Economía y negocios, 5(1), 33-40.

Polanyi, K. (2017 [1957]). La gran transformación (3. ${ }^{a}$ ed.), (G. Chailloux Laffita, Trad.) Fondo de Cultura Económica.

Santana Echeagaray, M. E. (2011). Recrear el dinero en una economía solidaria. (C. d. CISPo, Ed.) Polis [En línea], (29).

Schmidt, A. (2012). El concepto de naturaleza en Marx. Siglo xxi Editores.

Schuldt, J. (1997). Dineros alternativos para el desarrollo local. Centro de Investigación de la Universidad del Pacífico.

Shephard, D. (2011). Las monedas complementarias y la nueva realidad de las finanzas solidarias en países desarrollados y no desarrollados. Cayapa. Revista Venezolana de Economía Social, 11(21), 27-56.

Smith, A. (2008). Investigación sobre la naturaleza y causas de la riqueza de las naciones. Fondo de Cultura Económica. 
92 Revista Economía | II7 | mayo 202I

Toledo, V. (2013). El metabolismo social: una nueva teoría socioecológica. Relaciones. Estudios de historia y sociedad, 41-71. 